\title{
Stroke prevention in atrial fibrillation
}

\section{State of the art}

\author{
Li, Yan-Guang; Lip, Gregory Y H
}

Published in:

International Journal of Cardiology

DOI (link to publication from Publisher):

10.1016/j.ijcard.2018.09.057

Creative Commons License

CC BY-NC-ND 4.0

Publication date:

2019

Document Version

Accepted author manuscript, peer reviewed version

Link to publication from Aalborg University

Citation for published version (APA):

Li, Y-G., \& Lip, G. Y. H. (2019). Stroke prevention in atrial fibrillation: State of the art. International Journal of Cardiology, 287, 201-209. https://doi.org/10.1016/j.ijcard.2018.09.057

\section{General rights}

Copyright and moral rights for the publications made accessible in the public portal are retained by the authors and/or other copyright owners and it is a condition of accessing publications that users recognise and abide by the legal requirements associated with these rights.

- Users may download and print one copy of any publication from the public portal for the purpose of private study or research.

- You may not further distribute the material or use it for any profit-making activity or commercial gain

- You may freely distribute the URL identifying the publication in the public portal -

\section{Take down policy}

If you believe that this document breaches copyright please contact us at vbn@aub.aau.dk providing details, and we will remove access to the work immediately and investigate your claim. 


\section{Accepted Manuscript}

Stroke prevention in atrial fibrillation: State of the art

Yan-Guang Li, Gregory Y.H. Lip

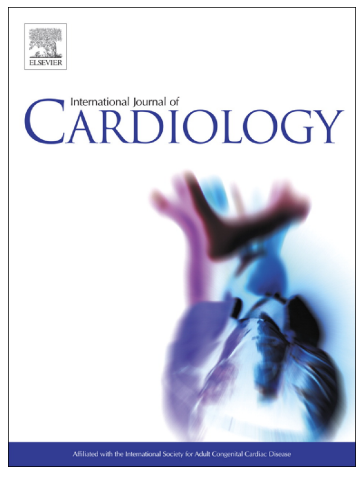

PII: $\quad$ S0167-5273(18)32178-8

DOI: $\quad$ doi:10.1016/j.ijcard.2018.09.057

Reference: $\quad$ IJCA 26978

To appear in: $\quad$ International Journal of Cardiology

Received date: $\quad 25$ July 2018

Revised date: $\quad 3$ September 2018

Accepted date: $\quad 17$ September 2018

Please cite this article as: Yan-Guang Li, Gregory Y.H. Lip, Stroke prevention in atrial fibrillation: State of the art. Ijca (2018), doi:10.1016/j.ijcard.2018.09.057

This is a PDF file of an unedited manuscript that has been accepted for publication. As a service to our customers we are providing this early version of the manuscript. The manuscript will undergo copyediting, typesetting, and review of the resulting proof before it is published in its final form. Please note that during the production process errors may be discovered which could affect the content, and all legal disclaimers that apply to the journal pertain. 


\section{Invited Review}

\section{Stroke Prevention in Atrial Fibrillation: State of the Art}

Yan-Guang Li (1,2), Gregory Y.H. Lip (1,2,3)

(1) Institute of Cardiovascular Sciences, University of Birmingham, and Liverpool Centre for Cardiovascular Science, University of Liverpool, United Kingdom; (2) Department of Cardiology, Chinese PLA Medical School, Chinese PLA General Hospital, Beijing, China; and (3) Aalborg Thrombosis Research Unit, Department of Clinical Medicine, Aalborg University, Aalborg, Denmark

\section{Corresponding Author}

Professor Gregory Y.H. Lip

Institute of Cardiovascular Sciences, University of Birmingham, Birmingham, England (UK)

Tel: +44 121 5075080; Email: g.y.h.lip@bham.ac.uk

\section{Declarations of interest}

None directly related to this paper

GYHL: Consultant for Bayer/Janssen, BMS/Pfizer, Medtronic, Boehringer Ingelheim, Novartis, Verseon and Daiichi-Sankyo. Speaker for Bayer, BMS/Pfizer, Medtronic, Boehringer Ingelheim, and Daiichi-Sankyo. No fees are directly received personally.

YGL receives funding from the China Scholarship Council (CSC) for his Ph.D. studies. 


\section{Abstract}

Stroke prevention is the cornerstone of the management of patients with atrial fibrillation (AF). Individual stroke risk stratification is generally the first step of deciding whether oral anticoagulation (OAC) will benefit patients with AF. Given that existing approaches to the prediction of 'high risk' subjects are of limited value, the initial focus should be the identification of 'low-risk' patients who do not need antithrombotic therapy. For this, the $\mathrm{CHA}_{2} \mathrm{DS}_{2}$-VASc score (congestive heart failure, hypertension, age $\geq 75$ [2 points], diabetes mellitus, previous stroke/transient ischemic attack [2 pints], vascular disease, age 65-74, female sex) performs well in identifying really low-risk patients (score of 0 in males or 1 in females), for whom OAC can be omitted.

The approach to AF management has changed, with the non-vitamin $\mathrm{K}$ antagonist oral anticoagulants (NOACs) providing relatively better efficacy, safety and convenience compared with the traditional vitamin $\mathrm{K}$ antagonists (VKAs). The latter drugs are performing well, if attention is directed towards good quality anticoagulation control, as reflected by a time in therapeutic range (TTR) $>70 \%$. Nevertheless, OAC use remains suboptimal especially in some regions, such as Asia and Africa. Long-term adherence and quality of OAC use need to be maintained for better outcomes in patients with AF.

Keywords: Atrial fibrillation, Stroke, Prevention, Risk, Anticoagulation, Integrate care 


\section{Introduction}

Atrial fibrillation (AF) is the most common arrhythmia, conferring a fivefold increased risk of stroke[1]. AF accounts for $15 \%$ of all ischemic strokes and $33 \%$ of strokes in the elderly[2]. Stroke events resulting from AF are more severe, associated with high risks of morbidity and mortality than those of non-AF etiologies[3]. Therefore, stroke prevention is an essential part of management for patients with AF.

The approach to stroke prevention for AF merits consideration of three steps. First, recognizing those patients at low risk of stroke for whom oral anticoagulation (OAC) can be omitted; second, choosing the right anticoagulant according to patient clinical profile; and third, optimizing the quality of anticoagulation control, especially if VKA (e.g. warfarin) are used, and attention to long-term adherence.

Compared to control or placebo, VKA reduces stroke by $64 \%$ and all-cause mortality by $26 \%$ [4]. In patients using VKA, quality of anticoagulation control is important, and a TTR $>70 \%$ should be maintained to improve outcomes[5]. The introduction of the non-vitamin K antagonist anticoagulants (NOACs), including factor II inhibitor, dabigatran, factor $\mathrm{X}$ inhibitors, rivaroxaban, apixaban and edoxaban, has changed the landscape of stroke prevention in AF patients worldwide, offering relatively improved efficacy, safety and convenience compared with the traditional VKAs[6-12].

Indeed, all NOACs are associated with a reduced risk for intracranial hemorrhage (ICH), even compared with well-controlled VKAs[13, 14]. In addition, NOAC-related bleeding events are less 
severe, especially for ICH[15]. The use of NOACs is associated with better treatment adherence compared with VKAs[16].

Nevertheless, OAC use remains suboptimal especially in some regions, such as Asia and Africa. Long-term adherence and quality of OAC use need to be maintained for a better outcome in patients with AF[17]. Significant barriers to OAC use still exist, and fear of bleeding events is the major reason for non-prescription in clinical practice. Indeed, OAC cessation and non-adherence is associated with poor outcomes[18, 19]. In general, clinicians have underestimated the benefits and overestimated bleeding risks in $\mathrm{OAC}$ use. Of note, aspirin does not reduce the risk of stroke in $\mathrm{AF}$ patients, but increases the risk of ICH, leading to a neutral or negative net clinical benefit (NCB)[20].

This narrative review aims to provide an overview of the evidence on stroke prevention for patients with AF. We provide an evidence-based pathway of stroke prevention in AF, signposting risk evaluation, OAC decision-making and anticoagulation quality control, with the aim of providing a simple and holistic approach for stroke prevention and integrated care for patients with AF (Figure 1).

\section{Stroke risk stratification and oral anticoagulation decision-making}

Stroke risk stratification is the first step in managing AF patients. The risk of stroke in AF is not homogenous and increases with the presence of more risk factors for stroke. Given that most patients with AF are at risk of stroke, initially ruling out those who are 'low-risk' would be a more effective 
way to direct stroke prevention, following which OAC can be offered to all patients with $\geq 1$ nongender stroke risk factors[21].

Patients with low risk of stroke are those with the $\mathrm{CHA}_{2} \mathrm{DS}_{2}$-VASc score of 0 in males or 1 in females[22]. In guidelines, patients with $\mathrm{CHA}_{2} \mathrm{DS}_{2}$-VASc score $\geq 2$ in males or $\geq 3$ in females are given a strong recommendation for $\mathrm{OAC}[23]$, while for patients with a single non-gender stroke risk factor (i.e. $\mathrm{CHA}_{2} \mathrm{DS}_{2}$-VASc score of 1 in males or 2 in females), stroke risks may vary with a particular risk factor. Overall, the use of OAC should still be considered given the positive net clinical benefit (NCB) in such 'medium-risk' patients[20, 24-31]. In general, the tipping point for obtaining an NCB is a stroke threshold of $1.7 \%$ year for VKA, and $0.9 \%$ for the NOACs[32].

As mentioned, for a single risk factor with same points, the stroke risk could be different. For example, AF patients at age of 65 and those at 74 have the same assigned 1 point for age, but the stroke risks are clearly different. Patients' stroke risk is continuous, while a score-based cutoff value is made arbitrary to facilitate simplicity and clinical practicality. Thus, some individualization is needed, for example, for an AF patient at 64 years and borderline hypertension (e.g. 139/80 mmHg) or a pre-diabetic stage, the increased stroke risk would best trigger OAC indication.

In the real-world setting, the stroke risk of patients with AF is not static. Increasing age, different blood pressure control, status of heart failure and incident risk factors would all contribute to a dynamic change in stroke risk. Thus, a patient without an identified stroke risk factor at baseline may need OAC during follow-up. Baseline risk assessment, even using the $\mathrm{CHA}_{2} \mathrm{DS}_{2}$-VASc score, may 
not necessarily relate to stroke risk in five or ten years later. For example, Chao et al studied 31,039 patients from Asia, and found the mean $\mathrm{CHA}_{2} \mathrm{DS}_{2}$-VASc score increased from 1.29 at baseline to 2.31 during follow-up[33]. The change in risk (i.e. 'delta $\mathrm{CHA}_{2} \mathrm{DS}_{2}-\mathrm{VASc}$ ') had predictivity for stroke events compared with baseline or follow-up $\mathrm{CHA}_{2} \mathrm{DS}_{2}$-VASc scores[33]. Therefore, regular review and reassessment of stroke risk is recommended in recent guidelines given the dynamic nature of stroke risk[34].

Age $\geq 65$ is a risk factor for stroke, but this age threshold has been derived from Western populations. Nevertheless, Asian populations have relatively lower age threshold for an increased stroke risk[35]. In a cohort study from Taiwan $(n=186,570)$, for example, patients could be further stratified into 2 subgroups with different stroke risk ( $>50$ years of age: 1.78\%/year vs. $<50$ years of age: $0.53 \% / y e a r)[35]$. Thus, age 50 to 74 years has been proposed with 1 point in the modified $\mathrm{CHA}_{2} \mathrm{DS}_{2^{-}}$ VASc score[36].

A recent study using three nationwide registries including 239,671 AF patients demonstrated that sex category (i.e. female sex) in the $\mathrm{CHA}_{2} \mathrm{DS}_{2}$-VASc score is a risk modifier rather than a risk factor[37]. Females exhibit a higher stroke risk with significant interaction with other risk factors, whereby females have a higher risk than males at age $\geq 65$ or with $\geq 1$ other risk factors[37].

\section{Beyond clinical risk stratification with established risk scores}

There are several biomarker-based scoring systems proposed to predict stroke and/or systemic embolism (SE) in patients with AF [38-40]. These scoring systems have included biomarkers based 
on blood tests or cardiac imaging, and have marginally better c-indexes compared with the $\mathrm{CHA}_{2} \mathrm{DS}_{2}$-VASc score. Nevertheless, scoring systems which need laboratory tests would reduce the convenience of a swift stroke risk evaluation in busy clinical settings, and delayed biomarker reading may defer OAC initiation. Also, significant heterogeneity of these biomarkers between populations and laboratory variability may make it difficult to define a cut-off value, especially since many of the studies proposing these biomarker-based scores were from highly selected anticoagulated clinical trial cohorts. Also, using a baseline biomarker level to predict stroke events years later may be unreasonable, given that patients get older and acquire incident risk factors. Second, some biomarkers, such as brain natriuretic peptide, are highly dependent on the patient's clinical situation, e.g. infection, water-sodium retention, etc.

Current risk scores do not consider AF subtypes, i.e., paroxysmal AF, persistent AF, permanent AF, as prior studies show that stroke risk is dependent on underlying risk factors rather than the arrhythmia subtype[21, 41-43]. Nevertheless, persistent AF may contribute higher stroke risk compared with paroxysmal AF[44, 45]. Indeed, in the non-anticoagulated AF patients, AF pattern was the second strongest predictor after prior stroke or transient ischemic attack (TIA) with an $83 \%$ and $44 \%$ increased risk for permanent and persistent AF compared with paroxysmal AF[45].

Furthermore, subclinical AF (SCAF) is associated with stroke risk in patients with cardiac implantable electronic device (CIED). For example, in the Asymptomatic Atrial Fibrillation and Stroke Evaluation in Pacemaker Patients and the Atrial Fibrillation Reduction Atrial Pacing Trial (ASSERT) trial $(\mathrm{n}=2,580)$, SCAF duration $>24 \mathrm{~h}$ was associated with a significant increased risk of 
subsequent stroke or SE (hazard ratio [HR] 3.24, [1.51-6.95])[46]. A large analysis from the Atrial Fibrillation Clopidogrel Trial with Irbesartan for Prevention of Vascular Events (ACTIVE-A) and Apixaban Versus Acetylsalicylic Acid to Prevent Stroke in Atrial Fibrillation Patients Who Have Failed or Are Unsuitable for Vitamin K Antagonist Treatment (AVERROES) trials suggested a clear gradient of increasing risk of stroke/SE from paroxysmal to persistent to permanent AF, whereby the annualized ischemic stroke rates were $2.1 \%, 3.0 \%$ and $4.2 \%$ respectively, with an HR of 1.83 (1.432.35) for permanent vs. paroxysmal AF and HR of 1.44 (1.05-1.98) for persistent vs. paroxysmal $\mathrm{AF}[45]$. However, we are dealing with wide heterogeneity and variability here, as an individual patient may have a short paroxysm during one monitoring period, but get a very long period of AF (or SCAF) in the next monitoring period.

AF occurring transiently with stress (AFOTS) presents frequently in the setting of acute illness, between $4 \%$ to $44 \%$ [47], and patients with AFOTS have an AF recurrence rate of $42 \%$ at 5 years[48]. In one study $(n=1,409)$, stroke risk (HR, 1.13 [0.82-1.57]) did not differ between patients with AFOTS and those with incident paroxysmal AF[48]. A large retrospective cohort study $(n=48,484)$ demonstrated that patients with resolved AF had a high risk of stroke/TIA compared with those without AF (relative risk [RR], 1.63 [1.46-1.83])[49].

\section{Left atrial appendage occlusion}


Evidence on left atrial appendage occlusion (LAAO) in stroke prevention is increasing, and offers an alternative to $\mathrm{OAC}$ among patients with contraindications for anticoagulation. Evidence for the use of LAAO comes from the WATCHMAN Left Atrial Appendage System for Embolic Protection in Patients with Atrial Fibrillation (PROTECT-AF) $(n=707)[50]$ and the Prospective Randomized Evaluation of the WATCHMAN Left Atrial Appendage Closure Device in Patients with Atrial Fibrillation vs. Long-term Warfarin Therapy (PREVAIL) $(\mathrm{n}=407)[51]$ trials. In their metaanalysis[52], ischemic stroke/SE rate was numerically higher with LAAO (HR, 1.71; p=0.080), but LAAO was associated with a lower risk of hemorrhagic stroke $(\mathrm{HR}, 0.20 ; \mathrm{p}=0.002)$ and similar major bleeding risk $(\mathrm{HR}, 0.91 ; \mathrm{p}=0.60)[52]$. In the PROTECT-AF study, procedural-related adverse events at 7 days after the procedure occurred in $8.7 \%$ of patients, including pericardial tamponade requiring intervention in 4.0\%[50]. In the Amulet Global Observational Registry $(n=1,088)$, major procedural complications occurred in $3.2 \%$ of patients, including pericardial effusion or tamponade in $1.2 \%$, vascular complication in $0.9 \%$, periprocedural stroke in $0.2 \%$, and death in $0.2 \%$ [53].

Currently, LAAO has a Grade IIb recommendation in the 2016 ESC guideline, and is limited to patients with high risk of bleeding who refuse to take OAC[23]. The performance of LAAO has never been compared against the NOACs in large randomized trials, which may provide more insights considering the safety advantages of NOACs over VKA, especially for ICH. One network meta-analysis indirectly found a trend towards higher rates of ischemic stroke with Watchman compared with NOACs (odds ratio [OR], 2.60 [0.60-13.96]) and a non-significant difference in of hemorrhagic stroke (OR, 0.44 [0.09-2.14])[54]. In addition, LAAO cannot eliminate the need for antithrombotic therapy, including the peri-procedural anticoagulation and long-term antiplatelet 
therapy, which may defeat the safety profile of LAAO.

\section{Unmet needs in oral anticoagulant use}

\section{Current trends}

Despite the well-documented efficacy and safety of OAC, an unmet OAC use remains common worldwide[55, 56]. In the last decade, OAC use remains low (approximately 50\%) among $\mathrm{AF}$ patients at high-risk of stroke[57]. Generally, OAC use in Western countries is better than in Asia.

In the EurObservational Research Programme-Atrial Fibrillation (EOPR-AF) registry from Europe in $2015(\mathrm{n}=10,000)$, OAC was used in 79.2\%[58]. In the Global Registry on Long-Term Oral Antithrombotic Treatment in Patients with Atrial Fibrillation (GLORIA-AF) registry including 17,162 patients with AF from Europe, $52.4 \%$ of the patients received NOAC, $5.7 \%$ received antiplatelet alone and only $4.1 \%$ remained untreated[59]. Similarly, the Antithrombotic Treatment Patterns in Patients with Newly Diagnosed Nonvalvular Atrial Fibrillation (GARFIELD-AF) registry involving recently diagnosed AF from 2010 to $2015(n=39,670)$ reported an increase in OAC use from $57.4 \%$ to $71.1 \%$, and a significant increase of NOACs use from $4.2 \%$ to $37.0 \%$, while antiplatelet monotherapy declined from $30.2 \%$ to $16.6 \%[60]$.

In Asia, suboptimal OAC use is more prevalent. For example, in a survey from China, involving 32 hospitals and 7,977 AF patients demonstrated OAC use of $36.5 \%$ among those with a $\mathrm{CHA}_{2} \mathrm{DS}_{2^{-}}$ VASc score $\geq 2$ between 2011 to 2014 , although this increased from $30.2 \%$ to $57.7 \%$ [61]. In AF 
patients from Korea between 2008 and $2015(n=276,246)$, OAC use increased from $34.7 \%$ to $50.6 \%$ and NOAC use accounted for $50 \%$ of total OAC use, while aspirin use consistently decreased from $48.2 \%$ to $31.5 \%[62]$.

\section{Quality of anticoagulation control}

Effective anticoagulation using VKA requires a TTR $\geq 65-70 \%$, but the TTR can be influenced by multiple factors, where the more common ones have been summarized in the $\mathrm{SAMeTT}_{2} \mathrm{R}_{2}$ score (sex, age, medical history, treatment of interaction medication, tobacco use, race $)^{4}$ [63]. A patient on VKA with a $\mathrm{SAMe}-\mathrm{TT}_{2} \mathrm{R}_{2}$ score $>2$ tends to have suboptimal TTR, who may need more frequent international normalized ratio (INR) checks, education/counselling on optimizing VKA use or switching instead to a NOAC. In general, the patients with AF from Western populations have higher TTR when compared with those from other populations. In the PREvention oF thromboembolic events-European Registry in Atrial Fibrillation (PREFER IN AF) registry, for example, the mean TTR ranged from $70.3 \%$ in Spain to $72.5 \%$ in the UK and $81.4 \%$ in Germany[64, 65]. The mean TTR has been reported as higher in Australia (82\%) than in Singapore (58\%)[66]. In Malaysia, the mean TTR was $65.1 \%$ in a professional anticoagulation clinic and only $48.3 \%$ in general clinics[67]. In Thailand, the mean TTR ranged from $40.1 \%$ to $62.7 \%$ in a cohort study with 433 AF patients[68]. In a survey from Saudi Arabia, the mean TTR was 59\%[69]. Also, in a cross-sectional study from South Africa, only $48.5 \%$ of patients achieved target therapeutic range[70]. In a subanalysis of the Effective Anticoagulation with Factor Xa Next Generation in Atrial Fibrillation-Thrombolysis in Myocardial Infarction 48 (ENGAGE AF-TIMI 48) trial $(n=21,105)$, the mean TTR was also low $(56 \%)$ in East Asia[71]. 


\section{Off-label reduced dosage of NOACs}

Despite the well-established efficacy and safety of NOACs, in everyday practice, clinicians are often more concerned about drug-related bleeding than ischemic stroke/SE, leading to a high rate of inappropriate underdosing of NOACs[72]. Barra et al. demonstrated that $57 \%$ of patients who were treated with a reduced dose of rivaroxaban did not meet the explicit dose reduction criteria in the label[73]. In the ORBIT-AF II registry (Outcomes Registry for Better Informed Treatment of Atrial Fibrillation phase II), $9.4 \%$ of patients received a reduced dose of NOACs, which was associated with increased cardiovascular hospitalization (HR, 1.26 [1.07-1.50])[74]. In a real-world study including 14,845 AF patients from the U.S., among 13,392 patients without renal indication for dose reduction, $13.3 \%$ received inappropriate reduced dose of NOACs; such underdosing was associated with a higher risk of stroke (HR, 4.87 [1.30-18.26]), but a non-significant difference in major bleeding [75]. In this study, patients with age $\geq 80$ had highest proportion of underdosing (apixaban $48.0 \%$, dabigatran $25.7 \%$, rivaroxaban $35.4 \%$ )[75]. Although a few studies showed that even lowdose NOACs had equivalent efficacy and reduced risk of major bleeding (RR, 0.61 [0.40-0.91]) compared to warfarin[76], clinicians should not be encouraged to use off-label dosages of NOACs, unless the presence of certain criteria justifying dose reduction - the evidence suggests that there is little safety advantage but more adverse outcomes result.

\section{Reversal of anticoagulant activity in urgent medical conditions}


Although the bleeding risk is low in NOACs, reversing the anticoagulation activity is important when individuals are suffering from serious bleeding, overdosing or require urgent surgery. In general, some supportive measures could be conducted, including mechanical/surgical management, volume resuscitation, blood transfusion, prothrombic complex concentrates injection, etc. For overdosed patients or those who ingested NOACs within 2 to 4 hours, oral activated charcoal may attenuate drug absorption. Hemodialysis is also an optional choice for patient taking dabigatran but not for those taking FXa inhibitors given the latter drugs are highly protein-bounded[77].

More important is that specific reversal agents are/coming to available in market, which effectiveness and safety in reversing anticoagulation activity. For example, idarucizumab is a specific antibody that binds all the dabigatran in plasma within minutes[78]. In the Reversal Effects of Idarucizumab on Active Dabigatran (RE-VERSE AD) study, idarucizumab rapidly (less than 10 minutes), durably, and safely reversed the anticoagulant effect of dabigatran[79].

Andexanet alpha is a recombinant modified human factor Xa decoy protein that has been shown to rapidly (within 2-5 minutes) and completely (>90\%) reverse the inhibition of factor $\mathrm{Xa}[80]$. The Andexanet Alfa for Acute Major Bleeding Associated with Factor Xa Inhibitors (ANNEXA) study is an ongoing, multicenter, prospective, open-label, single-group study investigating the role of andexanet in patients with acute major bleeding[81]. On the basis of descriptive preliminary analysis of this study, an initial bolus and subsequent 2-hour infusion of andexanet substantially reduced antifactor Xa, with effective hemostasis occurring in 79\%[81]. 


\section{Special patient subgroups}

OAC use in some subgroups of patients, e.g. those with high bleeding risk, renal dysfunction, recent ICH or very elderly patients, can be challenging. Clinicians should fit the right anticoagulants to patient's clinical profile, including those comorbidities such as acute coronary syndrome (ACS), concomitant antiplatelet use, peri-AF ablation procedure, cardioversion, chronic kidney disease (CKD) and elderly patients[14].

\section{High bleeding risk}

When initiating OAC, assessment of bleeding risk is necessary, which should be performed at each medical contact, considering the dynamic risk profile of AF patients who are often elderly with multiple comorbidities. Bleeding assessment can be done using currently available and validated bleeding risk scores, such as the HAS-BLED score (uncontrolled hypertension, abnormal renal/liver function, stroke, bleeding history or predisposition, labile International Normalized Ratio [INR], elderly, drugs/alcohol concomitantly) ${ }^{7}$. The HAS-BLED score is easy for clinical application with similar or better accuracy in predicting major bleeding events compared with other scoring systems which may be more complex and less user-friendly [82-85].

In 'real-world' settings, the individual risk of bleeding is not static. Indeed, among the components of the HAS-BLED score, there are modifiable risk factors, including uncontrolled hypertension, liver/renal function, anemia, labile INR and drugs/alcohol consumption. The changes or management of these risk factors would result in a different status of bleeding risk. Indeed, in a 'real-world' 
nationwide AF cohort $(n=19,566)$, follow-up HAS-BLED or 'delta HAS-BLED score' was more predictive of major bleeding compared with baseline HAS-BLED score[86].

A high bleeding risk does not eliminate the need for OAC. The HAS-BLED score and other scoring systems were designed to draw attention to modifiable bleeding risk factors[87-89]. and to 'flag up' patients with high bleeding risk, for whom more frequent follow-up, monitoring and education are needed[21]. While modifiable bleeding risk factors should be addressed in all patients, relying on this alone is an inferior strategy to an established bleeding risk score to assess bleeding risk[87, 90 , 91].

Very elderly patients

Evidence for OAC among very elderly patients, such as those over 80 or 90 , remains scarce as such patients were under-represented in clinical trials. These patients generally comorbid with multiple risk factors for stroke and bleeding, thus associating with high risks of both events. A meta-analysis $(n=8,932)$ show that the benefit of OAC increases with age, and the harm associated with aspirin increases[92]. Such a trend for benefit has also been observed in patients on NOAC[14]. Treatment of elderly patients with edoxaban provided a greater absolute reduction in bleeding events over warfarin compared to young patients[93]. A recent study from an Asian population, including 11,046 AF patients $>90$ years, showed that warfarin was associated with a lower risk of ischemic stroke and positive NCB[94]. Furthermore, NOACs were associated with a lower risk of ICH compared with the VKA (HR, 0.32[0.10-0.97])[94]. 


\section{Concomitant coronary artery disease (CAD)}

AF commonly coexists with CAD, either as stable CAD or ACS[95]. Thus, combination therapy of anticoagulant and antiplatelet agents is commonly used among patients with AF and CAD, which is associated with increased risk of bleeding (HR, 1.50 [1.23-1.82])[96]. Among AF patients experiencing ACS or undergoing PCI, triple therapy (OAC plus dual antiplatelets) should be used for the initial period, followed by OAC plus single antiplatelet drug and after 1 year, OAC alone[97].

The Randomized Evaluation of Dual Antithrombotic Therapy with Dabigatran versus Triple Therapy with Warfarin in Patients with Non-valvular Atrial Fibrillation Undergoing Percutaneous Coronary Intervention (REDUAL PCI) trial $(\mathrm{n}=2,725)$ showed lower risk of major or clinically relevant nonmajor bleeding with dabigatran plus $\mathrm{P} 2 \mathrm{Y} 12$ receptor inhibitor (110mg group: HR, 0.52 [0.420.63 and $150 \mathrm{mg}$ group: 0.72 [0.58-0.88] and no significant difference in stroke risk(110mg group: HR, 1.30 [0.63-2.67] and 150mg groups: 1.09 [0.42-2.83]), when compared with a warfarin-based triple therapy group[98]. In the Prevention of Bleeding in Patients with Atrial Fibrillation Undergoing PCI (PIONEER AF-PCI) trial (n=2124), the rivaroxaban group (rivaroxaban 15mg once daily plus single antiplatelet group and rivaroxaban $2.5 \mathrm{mg}$ twice daily plus dual antiplatelets group) had lower risk of bleeding (15mg group: HR, 0.59[0.47-0.76]; 2.5mg group: HR, 0.63[0.50-0.80]), compared with triple therapy with warfarin and similar risks of ischemic stroke (15mg group: HR, 1.07 [0.39-2.96]; 2.5mg group: HR, 1.36 [0.52-3.58])[99].

Recently, the 2018 Joint European Consensus Document on the Management of Antithrombotic Therapy in AF Patients Presenting with ACS and/or Undergoing PCI was published, providing an 
up-to-date evidence-based guidance on stroke prevention among AF patients with ACS and/or undergoing PCI, which is endorsed by HRS, APHRS, LAHRS and CASSA[100]. Other ongoing trials will provide new evidence. For example, the Apixaban Versus Warfarin in Patients with AF and ACS or PCI study (AUGUSTUS study, NCT02415400) is an open-label, $2 \times 2$ factorial, randomized, controlled non-inferiority clinical trial to evaluate the safety of apixaban vs. VKA and aspirin vs. aspirin placebo in patients with AF and ACS[101]. The Edoxaban Treatments vs. Vitamin K Antagonist in Patients with Atrial Fibrillation Undergoing Percutaneous Coronary Intervention (ENTRUST-AF-PCI) study is designed to evaluate the safety and to explore the efficacy of an edoxaban-based antithrombotic regimen vs. a VKA-based antithrombotic regimen in subjects with AF following PCI with stent placement[102].

\section{Chronic kidney disease}

The efficacy and safety of anticoagulation are closely related to renal function, especially for the NOACs. In patients with CKD $(n=48,500)$, warfarin use was associated with a $30 \%$ lower risk of stroke /SE and 35\% lower risk of death compared with no warfarin use[103]. In subgroup analysis, NOACs showed at least similar efficacy and better safety compared with warfarin in patients with mild or moderate CKD[14]; however, the excretion of the four NOACs also depends on renal function with a degree of renal excretion varying from $27-80 \%$ [104]. Of note, all the four NOACs randomised trials excluded patients with creatinine clearance $(\mathrm{CrCl})<30 \mathrm{~mL} / \mathrm{min}(<25 \mathrm{~mL} / \mathrm{min}$ for apixaban in the ARISTOTLE trial)[6, 105]. Therefore, assessing renal function in AF patients before or during taking NOAC is important to choose/modify the dosage of NOAC[106]. In the major NOACs trials, the assessment approach of renal function was with the Cockcroft-Gault Equation, 
which is well-validated and recommended in clinical practice to guide the dose of NOACs in CKD[107].

Currently, evidence on the use of $\mathrm{NOAC}$ in patients with $\mathrm{CrCl}<30 \mathrm{~mL} / \mathrm{min}$ comes from pharmacological modeling. In Europe, reduced dose rivaroxaban, apixaban and edoxaban are approved for thromboprophylaxis in patients with $\mathrm{CrCl} 15-29 \mathrm{~mL} / \mathrm{min}$ not for patients on dialysis. In the USA, the Food and Drug Administration approved the use of reduced-dose of dabigatran (75 mg twice daily) in $\mathrm{AF}$ patients with $\mathrm{CrCl} 15-29 \mathrm{~mL} / \mathrm{min}$, and in patients with end-stage renal disease on dialysis, apixaban (5 mg twice daily)[108]. Given that the $\mathrm{CHA}_{2} \mathrm{DS}_{2}$-VASc score is still a useful scheme to estimate the risk of stroke in AF patient with ESRD undergoing dialysis[109], a careful balancing of risk and benefit of NOACs treatment is possible.

\section{Peri-procedural AF ablation}

Anticoagulation during a peri-procedural period of AF ablation is important given the high inherent risk of stroke in this period. The major debate in this field focuses on several points, namely uninterrupted $\mathrm{OAC}$ use, bridging with heparin, OAC interruption/resumption and switching of OAC schemes. Indeed, bridging has been associated with a 4.5-fold increased risk of complications, including major pericardial effusion and major vascular events[110].

In the COMPARE (Role of Coumadin in Prevention Thromboembolism in AF Patients Undergoing Catheter Ablation) trial $(\mathrm{n}=1,584)$, interrupted anticoagulation significantly increased risk of thromboembolism $(4.94 \%$ vs. $0.25 \%, \mathrm{p}<0.001)$ compared with uninterrupted anticoagulation strategy, 
while major bleeding complications were non-significantly different $(0.76 \%$ vs. $0.38 \%, \mathrm{p}>0.05)$, and minor bleeding events were more frequent in the interrupted group $(22.0 \% v s .4 .1 \%, \mathrm{p}<0.001)[111]$. These results were confirmed by a meta-analysis including 12 studies and 17,434 patients, demonstrating that uninterrupted OAC was associated with lower risk of stroke/TIA $(0.25 \%$ vs. $1.21 \%, \mathrm{p}=0.003)$, stroke $(0.08 \%$ vs. $0.99 \%, \mathrm{p}<0.001)$, major bleeding $(1.44 \%$ vs. $2.03 \%, \mathrm{p}=0.02)$ and minor bleeding $(5.35 \%$ vs. $19.6 \%$; $\mathrm{p}<0.001)$ events[112]. Of note, NOACs have better safety and similar efficacy among patients receiving AF ablation, compared with the uninterrupted warfarin[10, $11,113-115]$.

Peri-procedural cardiac electronic devices implantation

One of the most severe complication after CIED implantation is the pocket hematoma. The Bridge or Continue Coumadin for Device Surgery Randomized Controlled Trial (BRUISE CONTROL) study, included 668 patients experiencing CIED implantation[116]. In this study, clinically significant hematoma was seen in $16 \%$ of the bridging group as compared to $3.5 \%$ of uninterrupted warfarin group[116]. Pooled analysis including 11,000 patients also demonstrated a lower risk of pocket hematoma in uninterrupted OAC as compared to those receiving bridging heparin therapy[117]. Considering the increasing usage of NOAC, the BRUISE-CONTROL-2 trial evaluated whether continued versus interrupted NOAC during peri-procedural period would reduce the risk of significant pocket hematoma[118]. The trial was stopped early due to futility, and both strategies had very low rates of device pocket hematoma. Thus, device surgery could be performed safely without interruption of NOACs. 
For clinical practice, warfarin could be continued with INR controlling between 2.0-3.0. In patients taking NOAC, anticoagulation may be discontinued prior to a planned procedure, and the time of discontinuation depends on the half-life of respective NOAC and patients' renal function. For patients with normal renal function, NOAC should be discontinued at least 24 hours preoperatively. For patients with renal dysfunction, a discontinuation for 48-72 hours seems reasonable. NOAC resumption is generally recommended within $24-48$ hours after the procedure.

\section{Peri-procedural cardioversion}

The 2016 ESC guideline recommends anticoagulation for $\geq 3$ weeks before elective cardioversion and at least 4 weeks thereafter in patients with AF episodes lasting over $48 \mathrm{~h}$ or unknown duration, regardless of stroke risk profile and types of cardioversion procedure[23]. The NOACs were noninferior to warfarin for stroke and bleeding events amongst AF patients undergoing cardioversion[119-121].

\section{Recent ischemic stroke}

Patients experiencing recent ischemic stroke events tend to have a high risk of recurrent ischemic stroke event and a tendency for hemorrhagic transformation. A previous meta-analysis demonstrated that immediate anticoagulation did not reduce the risk of stroke recurrence but associated with a higher risk of hemorrhagic transformation[122]. While early OAC resumption seems to reduce recurrent ischemic stroke, there was an increased risk of both intracranial and extracranial haemorrhage[123]. 
As recommended by the 2014 AHA/ASA guidelines, it seems reasonable to initiate OAC within 14 days for most stroke patients with $\mathrm{AF}$, and delay OAC for more than 14 days in those with high risk for hemorrhagic transformation, including large infarct, uncontrolled hypertension, hemorrhagic tendency and early hemorrhagic transformation showed by brain imaging[124]. Recently, a simple approach was proposed, i.e., the 1-3-6-12 day rule, which suggests that OAC initiation at 1 day after TIA; 3 days after a small, nondisabling infarct; 6 days after moderate stroke; and 12 days after a large infarct[125]; however, these suggestions are not supported by any trial evidence and are based on expert opinion. Given the significantly reduced ICH risk (up to 70\%) of NOAC[14], switching from traditional VKA to NOAC for patients experiencing recent stroke may provide better safety, which merits validation in randomized trials.

\section{Recent intracranial hemorrhage}

Given the lack of high-quality evidence, the most decision-making processes of OAC resumption are based on observational studies and empirical expert opinion. A large observation study $(\mathrm{n}=1,325)$ showed a non-significantly increased risk of ICH recurrence in patients restarted with warfarin compared with those without OAC resumption after ICH (HR, 1.31 [0.68-2.50])[126]. This was also confirmed by a recent meta-analysis which included 5,306 patients with ICH[127]. In contrast, OAC resumption is associated with a 51-66\% reduced risk of thromboembolism[126, 127].

In another large insurance-based study involving 12,917 AF patient with previous ICH, Chao TF et $a l$. found that the benefits of warfarin use are dependent on individual stroke risk (i.e. $\mathrm{CHA}_{2} \mathrm{DS}_{2^{-}}$ VASc score)[128]. In this study, among patients with a $\mathrm{CHA}_{2} \mathrm{DS}_{2}$-VASc score $\geq 6$, the number needed 
to treat for preventing 1 ischemic stroke was lower than the number needed to ham for producing 1 ICH with warfarin use (37 vs. 56); however, in patients with a $\mathrm{CHA}_{2} \mathrm{DS}_{2}$-VASc score $<6$, the number needed to treat was higher than the number needed to harm (63 vs. 53)[128].

The time of OAC resumption after $\mathrm{ICH}$ is an important factor for outcomes and it depends on individual. Normally, OAC could be resumed within 2-4 weeks after ICH, but delayed (>4 weeks) resumption should be considered for patients with extremely high risk of bleeding. For example, in patients with brainstem or cerebellar ICH, the timing could be delayed to at least 8-10 weeks after the event[129]. For AF patients with an extremely high $\mathrm{CHA}_{2} \mathrm{DS}_{2}$-VASc score (e.g. $>4$ ), an early OAC resumption seems reasonable after confirming the $\mathrm{ICH}$ is resolved.

\section{Patient-centered management}

Patient-centred approaches may help optimize management amongst patients with AF, with focus on patient values and preferences[130,131]. A shared decision-making process with patients regarding OAC use may improve anticoagulation management[132]. More recently, improved education and counseling could be reinforced by smart technology, such as apps on smartphones. For example, the pilot mAF App trial, which is the first prospective randomized trial of Mobile Health technology in patients with AF, showed that integrating clinical decision support, education and patients-involved management significantly improved drug adherence[133]. Also, a telemonitoring procedure leads to good adherence and could be improved with additional feedback from patients[134]. 
Integrated care

AF patients are often elderly with multiple comorbidities, and have a high risk of all-cause mortality and hospitalization. Indeed, nearly $70 \%$ of deaths are caused by cardiovascular disease[135], despite high rates of anticoagulation. Most deaths in AF are not related to stroke (which only accounts for 1 in 10 deaths), while 7 in 10 deaths are cardiovascular. Thus, a more integrated management strategy is needed. Such 'integrated care' in AF has been associated with a lowered risk of death and hospitalizations[136].

Integrated care can be simplified into the "Atrial Fibrillation Better care (ABC)" pathway, as follows: 'A' Avoid stroke with Anticoagulation; 'B' Better symptom management, involving patient-centred, symptom-directed decisions on rate or rhythm control; and 'C' Cardiovascular and other comorbidity risk management, including attention to lifestyle changes (obesity management, regular exercise, avoiding alcohol, etc.)[137] (Figure 1). Compliance with this ABC pathway approach has been shown to reduce mortality and other adverse events in anticoagulated patients with AF[138].

\section{Conclusion}

Stroke prevention in patients with AF is the cornerstone of management for this common arrhythmia. Current efforts will still focus on the following: (i) Improving the accuracy of identifying patients at low risk for thromboembolism; (ii) Exploring more effective and safer approaches to avoid stroke, 
based on a better understanding of the mechanism of stroke events in AF; and (iii) Increasing OAC use and patients' adherence, especially in many regions of the world, such as Asia, Africa and Middle East. Stroke prevention is merely one component of an integrated managing approach to AF, and attention to symptoms and cardiovascular or comorbidity management should also be emphasized. 


\section{FIGURE LEGENDS}

Figure 1. The "ABC" pathway and "Birmingham 3-step" for AF management and stroke prevention

NOAC=non-vitamin $\mathrm{K}$ antagonist anticoagulant; $\mathrm{OAC}=$ oral anticoagulant; $\mathrm{TTR}=$ time in therapeutic range; $\mathrm{VKA}=$ vitamin $\mathrm{K}$ antagonist. 


\section{References}

[1] Wolf PA, Abbott RD, Kannel WB. Atrial fibrillation as an independent risk factor for stroke: the Framingham Study. Stroke; a journal of cerebral circulation. 1991;22:983-8.

[2] Heart disease - Atrial fibrillation - Heart and Stroke Foundation of Canada http://www.heartandstroke.ca/heart/conditions/atrial-fibrillation. Accessed 19 Jun 2016.

[3] Henninger N, Goddeau RP, Jr., Karmarkar A, Helenius J, McManus DD. Atrial Fibrillation Is Associated With a Worse 90-Day Outcome Than Other Cardioembolic Stroke Subtypes. Stroke. 2016;47:1486-92.

[4] Hart RG, Pearce LA, Aguilar MI. Adjusted-dose warfarin versus aspirin for preventing stroke in patients with atrial fibrillation. Annals of internal medicine. 2007;147:590-2.

[5] Haas S, Ten Cate H, Accetta G, Angchaisuksiri P, Bassand JP, Camm AJ, et al. Quality of Vitamin K Antagonist Control and 1-Year Outcomes in Patients with Atrial Fibrillation: A Global Perspective from the GARFIELD-AF Registry. PloS one. 2016;11:e0164076.

[6] Granger. CB, Alexander. JH, McMurray. JJV, Lopes. RD, Hylek. EM, Hanna. M, et al. Apixaban versus Warfarin in Patients with Atrial Fibrillation-ARISTOTLE study. The New England journal of medicine. 2011;365:981-92.

[7] Giugliano RP, Ruff CT, Braunwald E, Murphy SA, Wiviott SD, Halperin JL, et al. Edoxaban versus warfarin in patients with atrial fibrillation. The New England journal of medicine. 2013;369:2093-104.

[8] Patel. MR, Mahaffey. KW, Garg. J, Pan. G, Singer. DE, Hacke. W, et al. Rivaroxaban versus warfarin in nonvalvular atrial fibrillation. The New England journal of medicine. 2011;365:883-91. [9] Connolly. SJ, Ezekowitz. MD, Ch.B., Yusuf. S, Eikelboom. J, Parekh. A. Dabigatran versus 
warfarin in patients with atrial fibrillation. New England Journal of Medicine. 2009;361:1139-51.

[10] Wu S, Yang YM, Zhu J, Wan HB, Wang J, Zhang H, et al. Meta-Analysis of Efficacy and Safety of New Oral Anticoagulants Compared With Uninterrupted Vitamin K Antagonists in Patients Undergoing Catheter Ablation for Atrial Fibrillation. The American journal of cardiology. 2016;117:926-34.

[11] Cardoso R, Knijnik L, Bhonsale A, Miller J, Nasi G, Rivera M, et al. An updated meta-analysis of novel oral anticoagulants versus vitamin $\mathrm{K}$ antagonists for uninterrupted anticoagulation in atrial fibrillation catheter ablation. Heart rhythm : the official journal of the Heart Rhythm Society. 2018;15:107-15.

[12] Ntaios G, Papavasileiou V, Makaritsis K, Vemmos K, Michel P, Lip GYH. Real-World Setting Comparison of Nonvitamin-K Antagonist Oral Anticoagulants Versus Vitamin-K Antagonists for Stroke Prevention in Atrial Fibrillation: A Systematic Review and Meta-Analysis. Stroke. 2017;48:2494-503.

[13] Ruff CT, Giugliano RP, Braunwald E, Hoffman EB, Deenadayalu N, Ezekowitz MD, et al. Comparison of the efficacy and safety of new oral anticoagulants with warfarin in patients with atrial fibrillation: a meta-analysis of randomised trials. Lancet (London, England). 2014;383:955-62.

[14] Li YG, Pastori D, Lip GYH. Fitting the right non-vitamin K antagonist oral anticoagulant to the right patient with non-valvular atrial fibrillation: an evidence-based choice. Ann Med. 2018;50:1-15.

[15] Inohara T, Xian Y, Liang L, Matsouaka RA, Saver JL, Smith EE, et al. Association of Intracerebral Hemorrhage Among Patients Taking Non-Vitamin K Antagonist vs Vitamin K Antagonist Oral Anticoagulants With In-Hospital Mortality. JAMA. 2018;319:463-73.

[16] Gorst-Rasmussen A, Skjoth F, Larsen TB, Rasmussen LH, Lip GY, Lane DA. Dabigatran 
adherence in atrial fibrillation patients during the first year after diagnosis: a nationwide cohort study. J Thromb Haemost. 2015;13:495-504.

[17] Raparelli V, Proietti M, Cangemi R, Lip GY, Lane DA, Basili S. Adherence to oral anticoagulant therapy in patients with atrial fibrillation. Focus on non-vitamin $\mathrm{K}$ antagonist oral anticoagulants. Thrombosis and haemostasis. 2017;117:209-18.

[18] Rivera-Caravaca JM, Roldan V, Esteve-Pastor MA, Valdes M, Vicente V, Lip GYH, et al. Cessation of oral anticoagulation is an important risk factor for stroke and mortality in atrial fibrillation patients. Thrombosis and haemostasis. 2017;117:1448-54.

[19] Mazurek M, Shantsila E, Lane DA, Wolff A, Proietti M, Lip GYH. Guideline-Adherent Antithrombotic Treatment Improves Outcomes in Patients With Atrial Fibrillation: Insights From the Community-Based Darlington Atrial Fibrillation Registry. Mayo Clinic proceedings. 2017;92:120313. [20] Lip GY, Skjoth F, Nielsen PB, Larsen TB. Non-valvular atrial fibrillation patients with none or one additional risk factor of the CHA2DS2-VASc score. A comprehensive net clinical benefit analysis for warfarin, aspirin, or no therapy. Thromb Haemost. 2015;114:826-34.

[21] Lip G, Freedman B, De Caterina R, Potpara TS. Stroke prevention in atrial fibrillation: Past, present and future. Comparing the guidelines and practical decision-making. Thromb Haemost. 2017;117:1230-9.

[22] Kirchhof P, Benussi S, Kotecha D, Ahlsson A, Atar D, Casadei B, et al. 2016 ESC Guidelines for the management of atrial fibrillation developed in collaboration with EACTS. Eur Heart J. 2016;37:2893-962.

[23] Kirchhof P, Benussi S, Kotecha D, Ahlsson A, Atar D, Casadei B, et al. 2016 ESC Guidelines 
for the management of atrial fibrillation developed in collaboration with EACTS. Eur Heart J. 2016;37:2893-962.

[24] Joundi RA, Cipriano LE, Sposato LA, Saposnik G, Stroke Outcomes Research Working G. Ischemic Stroke Risk in Patients With Atrial Fibrillation and CHA2DS2-VASc Score of 1: Systematic Review and Meta-Analysis. Stroke. 2016;47:1364-7.

[25] Fauchier L, Lecoq C, Clementy N, Bernard A, Angoulvant D, Ivanes F, et al. Oral Anticoagulation and the Risk of Stroke or Death in Patients With Atrial Fibrillation and One Additional Stroke Risk Factor: The Loire Valley Atrial Fibrillation Project. Chest. 2016;149:960-8. [26] Lip GY, Skjoth F, Rasmussen LH, Larsen TB. Oral anticoagulation, aspirin, or no therapy in patients with nonvalvular AF with 0 or 1 stroke risk factor based on the CHA2DS2-VASc score. Journal of the American College of Cardiology. 2015;65:1385-94.

[27] Chao TF, Liu CJ, Wang KL, Lin YJ, Chang SL, Lo LW, et al. Should atrial fibrillation patients with 1 additional risk factor of the CHA2DS2-VASc score (beyond sex) receive oral anticoagulation? Journal of the American College of Cardiology. 2015;65:635-42.

[28] Olesen JB, Lip GY, Hansen ML, Hansen PR, Tolstrup JS, Lindhardsen J, et al. Validation of risk stratification schemes for predicting stroke and thromboembolism in patients with atrial fibrillation: nationwide cohort study. BMJ (Clinical research ed). 2011;342:d124.

[29] Lip GY, Nielsen PB. Should Patients With Atrial Fibrillation and 1 Stroke Risk Factor (CHA2DS2-VASc Score 1 in Men, 2 in Women) Be Anticoagulated? Yes: Even 1 Stroke Risk Factor Confers a Real Risk of Stroke. Circulation. 2016;133:1498-503; discussion 503.

[30] Hung Y, Chao TF, Liu CJ, Tuan TC, Lin YJ, Chang SL, et al. Is an Oral Anticoagulant Necessary for Young Atrial Fibrillation Patients With a CHA2DS2-VASc Score of 1 (Men) or 2 
(Women)? Journal of the American Heart Association. 2016;5.

[31] Fauchier L, Clementy N, Bisson A, Ivanes F, Angoulvant D, Babuty D, et al. Should Atrial Fibrillation Patients With Only 1 Nongender-Related CHA2DS2-VASc Risk Factor Be Anticoagulated? Stroke. 2016;47:1831-6.

[32] Eckman MH, Singer DE, Rosand J, Greenberg SM. Moving the tipping point: the decision to anticoagulate patients with atrial fibrillation. Circulation Cardiovascular quality and outcomes. 2011;4:14-21.

[33] Chao TF, Lip GYH, Liu CJ, Lin YJ, Chang SL, Lo LW, et al. Relationship of Aging and Incident Comorbidities to Stroke Risk in Patients With Atrial Fibrillation. Journal of the American College of Cardiology. 2018;71:122-32.

[34] Lip GYH, Banerjee A, Boriani G, Chiang Ce, Fargo R, Freedman B, et al. Antithrombotic Therapy for Atrial Fibrillation: CHEST Guideline and Expert Panel Report. Chest. 2018:https://doi.org/10.1016/j.chest.2018.07.040.

[35] Chao TF, Wang KL, Liu CJ, Lin YJ, Chang SL, Lo LW, et al. Age Threshold for Increased Stroke Risk Among Patients With Atrial Fibrillation: A Nationwide Cohort Study From Taiwan. Journal of the American College of Cardiology. 2015;66:1339-47.

[36] Chao TF, Lip GY, Liu CJ, Tuan TC, Chen SJ, Wang KL, et al. Validation of a Modified CHA2DS2-VASc Score for Stroke Risk Stratification in Asian Patients With Atrial Fibrillation: A Nationwide Cohort Study. Stroke. 2016;47:2462-9.

[37] Nielsen PB, Skjoth F, Overvad TF, Larsen TB, Lip GYH. Female Sex Is a Risk Modifier Rather Than a Risk Factor for Stroke in Atrial Fibrillation: Should We Use a CHA2DS2-VA Score Rather Than CHA2DS2-VASc? Circulation. 2018;137:832-40. 
[38] Ruff CT, Giugliano RP, Braunwald E, Murphy SA, Brown K, Jarolim P, et al. Cardiovascular Biomarker Score and Clinical Outcomes in Patients With Atrial Fibrillation: A Subanalysis of the ENGAGE AF-TIMI 48 Randomized Clinical Trial. JAMA Cardiol. 2016;1:999-1006.

[39] Hijazi Z, Lindback J, Alexander JH, Hanna M, Held C, Hylek EM, et al. The ABC (age, biomarkers, clinical history) stroke risk score: a biomarker-based risk score for predicting stroke in atrial fibrillation. European heart journal. 2016;37:1582-90.

[40] Hippisley-Cox J, Coupland C, Brindle P. Derivation and validation of QStroke score for predicting risk of ischaemic stroke in primary care and comparison with other risk scores: a prospective open cohort study. BMJ. 2013;346:f2573.

[41] Hohnloser SH, Pajitnev D, Pogue J, Healey JS, Pfeffer MA, Yusuf S, et al. Incidence of stroke in paroxysmal versus sustained atrial fibrillation in patients taking oral anticoagulation or combined antiplatelet therapy: an ACTIVE W Substudy. Journal of the American College of Cardiology. 2007;50:2156-61.

[42] Friberg L, Hammar N, Rosenqvist M. Stroke in paroxysmal atrial fibrillation: report from the Stockholm Cohort of Atrial Fibrillation. Eur Heart J. 2010;31:967-75.

[43] Hart RG, Pearce LA, Rothbart RM, McAnulty JH, Asinger RW, Halperin JL. Stroke with intermittent atrial fibrillation: incidence and predictors during aspirin therapy. Stroke Prevention in Atrial Fibrillation Investigators. Journal of the American College of Cardiology. 2000;35:183-7.

[44] Steinberg BA, Hellkamp AS, Lokhnygina Y, Patel MR, Breithardt G, Hankey GJ, et al. Higher risk of death and stroke in patients with persistent vs. paroxysmal atrial fibrillation: results from the ROCKET-AF Trial. Eur Heart J. 2015;36:288-96.

[45] Vanassche T, Lauw MN, Eikelboom JW, Healey JS, Hart RG, Alings M, et al. Risk of ischaemic 
stroke according to pattern of atrial fibrillation: analysis of 6563 aspirin-treated patients in ACTIVEA and AVERROES. European heart journal. 2015;36:281-7a.

[46] Van Gelder IC, Healey JS, Crijns H, Wang J, Hohnloser SH, Gold MR, et al. Duration of devicedetected subclinical atrial fibrillation and occurrence of stroke in ASSERT. European heart journal. 2017;38:1339-44.

[47] McIntyre WF, Healey J. Stroke Prevention for Patients with Atrial Fibrillation: Beyond the Guidelines. J Atr Fibrillation. 2017;9:1475.

[48] Lubitz SA, Yin X, Rienstra M, Schnabel RB, Walkey AJ, Magnani JW, et al. Long-term outcomes of secondary atrial fibrillation in the community: the Framingham Heart Study. Circulation. 2015;131:1648-55.

[49] Adderley NJ, Nirantharakumar K, Marshall T. Risk of stroke and transient ischaemic attack in patients with a diagnosis of resolved atrial fibrillation: retrospective cohort studies. BMJ (Clinical research ed). 2018;361:k1717.

[50] Holmes DR, Reddy VY, Turi ZG, Doshi SK, Sievert H, Buchbinder M, et al. Percutaneous closure of the left atrial appendage versus warfarin therapy for prevention of stroke in patients with atrial fibrillation: a randomised non-inferiority trial. Lancet. 2009;374:534-42.

[51] Holmes DR, Jr., Kar S, Price MJ, Whisenant B, Sievert H, Doshi SK, et al. Prospective randomized evaluation of the Watchman Left Atrial Appendage Closure device in patients with atrial fibrillation versus long-term warfarin therapy: the PREVAIL trial. Journal of the American College of Cardiology. 2014;64:1-12.

[52] Reddy VY, Doshi SK, Kar S, Gibson DN, Price MJ, Huber K, et al. 5-Year Outcomes After Left Atrial Appendage Closure: From the PREVAIL and PROTECT AF Trials. Journal of the American 
College of Cardiology. 2017;70:2964-75.

[53] Landmesser U, Schmidt B, Nielsen-Kudsk JE, Lam SCC, Park JW, Tarantini G, et al. Left atrial appendage occlusion with the AMPLATZER Amulet device: periprocedural and early clinical/echocardiographic data from a global prospective observational study. EuroIntervention. 2017;13:867-76.

[54] Koifman E, Lipinski MJ, Escarcega RO, Didier R, Kiramijyan S, Torguson R, et al. Comparison of Watchman device with new oral anti-coagulants in patients with atrial fibrillation: A network meta-analysis. International journal of cardiology. 2016;205:17-22.

[55] Mazurek M, Huisman MV, Rothman KJ, Paquette M, Teutsch C, Diener HC, et al. Regional Differences in Antithrombotic Treatment for Atrial Fibrillation: Insights from the GLORIA-AF Phase II Registry. Thromb Haemost. 2017;117:2376-88.

[56] Senoo K, An Y, Ogawa H, Lane DA, Wolff A, Shantsila E, et al. Stroke and death in elderly patients with atrial fibrillation in Japan compared with the United Kingdom. Heart. 2016;102:187882.

[57] Ogilvie IM, Newton N, Welner SA, Cowell W, Lip GY. Underuse of oral anticoagulants in atrial fibrillation: a systematic review. The American journal of medicine. 2010;123:638-45.e4.

[58] Huisman MV, Rothman KJ, Paquette M, Teutsch C, Diener HC, Dubner SJ, et al. Antithrombotic Treatment Patterns in Patients with Newly Diagnosed Nonvalvular Atrial Fibrillation: The GLORIA-AF Registry, Phase II. The American journal of medicine. 2015;128:1306-13 e1. [59] Bassand JP, Accetta G, Camm AJ, Cools F, Fitzmaurice DA, Fox KA, et al. Two-year outcomes of patients with newly diagnosed atrial fibrillation: results from GARFIELD-AF. Eur Heart J. 2016;37:2882-9. 
[60] Camm AJ, Accetta G, Ambrosio G, Atar D, Bassand JP, Berge E, et al. Evolving antithrombotic treatment patterns for patients with newly diagnosed atrial fibrillation. Heart (British Cardiac Society). 2017;103:307-14.

[61] Chang SS, Dong JZ, Ma CS, Du X, Wu JH, Tang RB, et al. Current Status and Time Trends of Oral Anticoagulation Use Among Chinese Patients With Nonvalvular Atrial Fibrillation: The Chinese Atrial Fibrillation Registry Study. Stroke. 2016;47:1803-10.

[62] Lee SR, Choi EK, Han KD, Cha MJ, Oh S, Lip GYH. Temporal trends of antithrombotic therapy for stroke prevention in Korean patients with non-valvular atrial fibrillation in the era of nonvitamin $\mathrm{K}$ antagonist oral anticoagulants: A nationwide population-based study. PloS one. 2017;12:e0189495.

[63] Apostolakis S, Sullivan RM, Olshansky B, Lip GYH. Factors affecting quality of anticoagulation control among patients with atrial fibrillation on warfarin: the SAMe-TT(2)R(2) score. Chest. 2013;144:1555-63.

[64] Le Heuzey JY, Ammentorp B, Darius H, De Caterina R, Schilling RJ, Schmitt J, et al. Differences among western European countries in anticoagulation management of atrial fibrillation. Data from the PREFER IN AF registry. Thromb Haemost. 2014;111:833-41.

[65] Ingram SJ, Kirkdale CL, Williams S, Hartley E, Wintle S, Sefton V, et al. Moving anticoagulation initiation and monitoring services into the community: evaluation of the Brighton and hove community pharmacy service. BMC Health Serv Res. 2018;18:91.

[66] Bernaitis N, Ching CK, Teo SC, Chen L, Badrick T, Davey AK, et al. Factors influencing warfarin control in Australia and Singapore. Thrombosis research. 2017;157:120-5.

[67] Thanimalai S, Shafie AA, Hassali MA, Sinnadurai J. Comparing effectiveness of two 
anticoagulation management models in a Malaysian tertiary hospital. Int J Clin Pharm. 2013;35:73643.

[68] Saokaew S, Sapoo U, Nathisuwan S, Chaiyakunapruk N, Permsuwan U. Anticoagulation control of pharmacist-managed collaborative care versus usual care in Thailand. Int J Clin Pharm. 2012;34:105-12.

[69] Alyousif SM, Alsaileek AA. Quality of anticoagulation control among patients with atrial fibrillation: An experience of a tertiary care center in Saudi Arabia. J Saudi Heart Assoc. 2016;28:239-43.

[70] Sonuga BO, Hellenberg DA, Cupido CS, Jaeger C. Profile and anticoagulation outcomes of patients on warfarin therapy in an urban hospital in Cape Town, South Africa. Afr J Prim Health Care Fam Med. 2016;8:e1-8. 
Figure 1

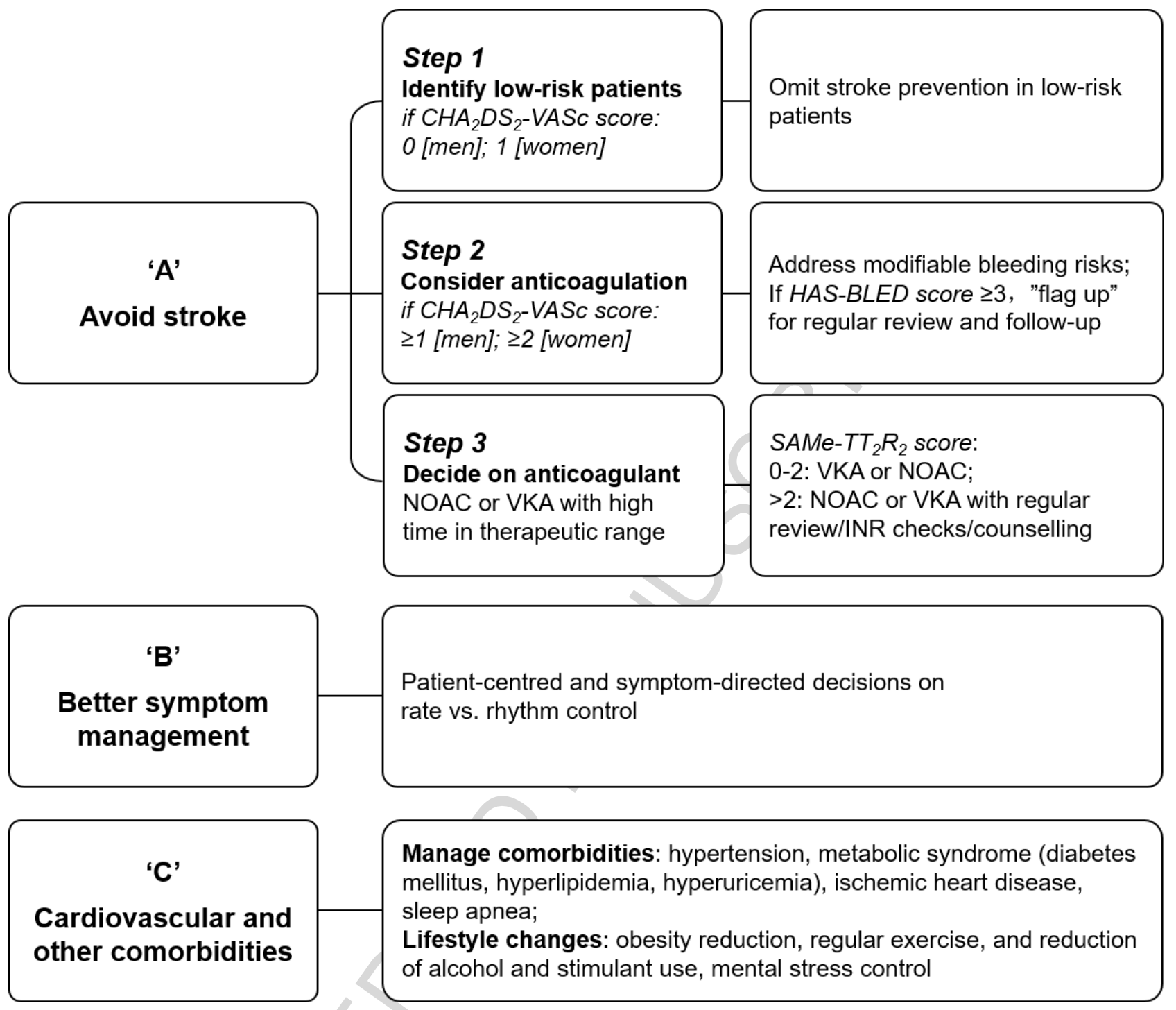




\section{Highlights:}

(i) Stroke prevention in patients with AF should initially focus on identifying low-risk group for which anticoagulation could be omitted;

(ii) In everyday clinical practice, anticoagulation decisions should be based on - but not limited to the established stroke risk scores; while individualization and attention to patient values and preferences is necessary;

(iii) Unmet needs in anticoagulation use are prevalent, especially among Asian and Africa populations; quality control for anticoagulation is important which has impact on outcomes. 